\section{Dissecting Suicidality Using a Combined Genomic and Clinical Approach}

'Never give in, never give in, never, never, never.'

- Winston Churchill

One person dies by suicide every $40 \mathrm{~s}$ worldwide, despite suicide being a potentially preventable tragedy. It is clear that better assessment, tracking, and prediction of suicidality (ideation, actions) are needed. We endeavored to develop better clinical data tools and objective blood biomarkers for such purposes. The two approaches are complementary, synergistic, and can inform each other.

First, in terms of clinical data, we developed a simple polyphenic risk score, based on phenes (phenotypic items) that are known risk factors for suicide. Similar to polygenic scoring, these were scored in a binary fashion as 0 or 1 (absent or present). This questionnaire/app, known as Convergent Functional Information for Suicide (CFI-S), has 22 easy-to-answer items, related to life issues, mental health, physical health, environmental stress, addictions, and cultural factors. It purposefully does not ask about suicidal ideation, as that is a loaded question in most settings, and because people who truly want to kill themselves may not share that information for fear of being stopped. CFI-S can be self-scored or clinician scored, obtained from patient interview, or from information from next of kin or medical records. CFI-S has shown good predictive ability for suicidal ideation and for future hospitalizations for suicidality, in men (Niculescu et al, 2015) and in women (Levey et al, 2016). It can have a therapy-guiding component, in that over half of the risk factors it identifies are correctable. In addition, we have developed and used a simple visual analog scale type of questionnaire/app, known as Simple
Affective State Scale (SASS), that provides a score for anxiety and one for mood at a particular moment in time (Niculescu et al, 2015; Levey et al, 2016). High anxiety scores and low mood scores were predictive for suicidal ideation and future hospitalizations by themselves, and even more so when combined with the CFI-S score, which provides broader contextual information.

Second, as the brain (the target organ) cannot be biopsied in live individuals, we endeavored to develop a liquid biopsy, ie, to find blood biomarkers for suicidality that would have translational and practical clinical utility. The possibility of finding blood biomarkers for psychiatric phenotypes had been substantiated by earlier pilot studies by our group focused on mood (Le-Niculescu et al, 2009), psychosis (Kurian et al, 2011), and suicide (Le-Niculescu et al, 2013). How and why peripheral molecular changes reflect what is happening in the brain is an area of ongoing research. Brain cells and blood cells share some genetic polymorphisms, as well as exposure to the internal milieu and whole-body responses such as stress. There are also more direct neuro-immune connections. However, it is likely that only a small proportion of peripheral changes in gene expression are relevant to and/ or concordant with brain changes related to suicide. Finding the true signal among all the noise is key to success. It is predicated on having, for example, a powerful longitudinal within-subject design to conduct the discovery, prioritization of findings using convergent functional genomics, and validation of the biomarkers in relevant independent cohorts (Niculescu et al, 2015). Using such approaches, we were successful in finding blood biomarkers that were predictive of suicidality, at least in the psychiatric patient populations that we have studied so far (Niculescu et al, 2015; Levey et al, 2016; Le-Niculescu et al, 2013). Some of them have prior brain gene expression evidence (Niculescu et al, 2015; Levey et al, 2016), which is part of our convergent approach to prioritize them. While the biomarkers were reasonably predictive by themselves, they were in general less predictive than the clinical information scores we described above. The biomarkers may serve a useful purpose when clinical information is not available and/or to (1) provide a window into the biology of suicidality, (2) help stratify patients, and (3) monitor disease course and response to treatment in an objective fashion. Importantly, when combined, the biomarker scores and clinical information scores were synergistic in terms of improved predictive ability (Niculescu et al, 2015; Levey et al, 2016).

It has not escaped our attention that the general strategy outlined here can be used to try to understand, predict, and improve other behavioral phenotypes.

\section{FUNDING AND DISCLOSURE}

This work was supported by an NIH Directors' New Innovator Award (1DP2OD007363) and a VA Merit Award (2I01CX000139) to ABN. ABN is listed as inventor on a patent application being filed by Indiana University, and is a co-founder of MindX Sciences. HL-N declares no conflict of interest.

\section{Alexander B Niculescu ${ }^{1,2,3}$ and Helen Le-Niculescu ${ }^{1}$ \\ ${ }^{1}$ Department of Psychiatry, Indiana University School of Medicine, Indianapolis, IN, USA; ${ }^{2}$ Stark \\ Neuroscience Research Institute, Indiana University School of Medicine, Indianapolis, IN, USA; ${ }^{3}$ Indianapolis VA Medical Center, Indianapolis, IN, USA \\ E-mail: anicules@iupui.edu}

Kurian SM, Le-Niculescu H, Patel SD, Bertram D, Davis J, Dike C et al (2011). Identification of blood biomarkers for psychosis using convergent functional genomics. Mol Psychiatry 16: 37-58.

Le-Niculescu H, Kurian SM, Yehyawi N, Dike C, Patel $\mathrm{SD}$, Edenberg HJ et al (2009). Identifying blood biomarkers for mood disorders using convergent functional genomics. Mol Psychiatry 14: 156-174. Le-Niculescu H, Levey DF, Ayalew M, Palmer L, Gavrin LM, Jain N et al (2013). Discovery and 
validation of blood biomarkers for suicidality. $\mathrm{Mol}$ Psychiatry 18: 1249-1264.

Levey DF, Niculescu EM, Le-Niculescu H, Dainton HL, Phalen PL, Ladd TB et al (2016). Towards understanding and predicting suicidality in women: biomarkers and clinical risk assessment. $\mathrm{Mol}$ Psychiatry 21: 768-785.

Niculescu AB, Levey D, Le-Niculescu H, Niculescu E, Kurian SM, Salomon D (2015). Psychiatric blood biomarkers: avoiding jumping to premature negative or positive conclusions. Mol Psychiatry 20: 286-288.

Niculescu AB, Levey DF, Phalen PL, Le-Niculescu H, Dainton HD, Jain N et al (2015). Understanding and predicting suicidality using a combined genomic and clinical risk assessment approach. Mol Psychiatry 20: 1266-1285.

Neuropsychopharmacology Reviews (2017) 42, 360-361; doi: 10.1038/npp.2016.228

\section{Food Addiction and Obesity}

The obesity epidemic in the US and abroad has increased interest in neurobiological mechanisms that contribute to eating in the absence of energy demand or hunger (overeating). Overeating, sometimes called 'hedonic' feeding because foods consumed are usually palatable and high in sugars and fats, is the leading cause of overweight and obesity in the US. One important factor that contributes to obesity is overeating triggered by sights, sounds, and smells (food cues) associated with palatable food (Stice et al, 2013; Tang et al, 2012). For example, obese and overweight people report greater food craving and consume larger portions in response to food cues. A wealth of preclinical studies have demonstrated that these cue-triggered motivational responses are mediated by brain mesocorticolimbic circuits, particularly dopamine and glutamate transmission within the nucleus accumbens (NAc). In humans, the magnitude of activations in the NAc in response to food cues predicts future weight gain in normal-weight people and inability to lose weight after obesity onset. This suggests that differences in NAc function may mediate enhanced cue-triggered urges to eat in susceptible individuals both before and after weight gain. The overlap in the neural systems involved and similarities between responses to food, addictive drugs, and cues associated with them have sparked vibrant discussion about 'food addiction' (Carter et al, 2016), despite some obvious differences between food and drug (eg, food does not have psychoactive properties in the pharmacological sense, nor is there a clear doseresponse relationship, etc.). So, can overeating that produces common obesity really be considered a form of addiction?

In a recent preclinical study we found that, prior to any diet manipulation, those rats that show the strongest motivational responses to a food cue subsequently gained the most weight when given free access to a fatty, sugary, 'junk-food' diet (Robinson et al, 2015). Obesity-prone rats are also more sensitive to the locomotoractivating effects of cocaine, indicating enhanced responsivity of mesolimbic systems, and have greater excitability of medium spiny neurons in the NAc core prior to the development of obesity (Oginsky et al, 2016a, b). Taken together, our data suggest that preexisting enhancements in NAc responsivity may render obesity-susceptible individuals more sensitive to the motivational properties of food cues. In addition, consumption of fatty, sugary foods alters NAc function in ways that also vary by susceptibility to obesity. For example, consumption of junk food enhances NAc glutamatergic transmission and produces an upregulation of calcium-permeable AMPA receptors (CP-AMPARs) in obesity-susceptible rats (Brown et al, 2015; Oginsky et al, 2016a). Interestingly, increases in CPAMPARs were found prior to obesity onset, suggesting that they may drive enhanced motivational responses that promote weight gain (see Oginsky et al, 2016b for further discussion).

Enhanced cue triggered motivation and accompanying increases in NAc responsivity are thought to underlie drug addiction (Flagel et al, 2009), and NAc CP-AMPARs mediate enhanced cue-trigged cocaine-seeking (Wolf, 2016). One pressing question that arises, however, is the extent to which the enhanced neurobehavioral responsivity found in obesitysusceptible rats is a form of aberrant motivation, akin to drug addiction. Brain reward circuits evolved in part to direct behavior towards food. Thus, enhancements in NAc responsivity and cue-triggered motivation could be described as an improved function of this system (ie, rendering individuals better able to find food) rather than dysfunction. Of course, not being able to curb cue-triggered urges in the current calorie-dense food environment is unhealthy, and it is not entirely clear where this idea leaves obesity-resistant individuals. Are they the outliers? However, to develop better prevention strategies for obesity it will be important to differentiate 'normal' neurobehavioral responses from fundamentally aberrant ones and to continue to challenge ourselves by asking these questions.

\section{FUNDING AND DISCLOSURE}

Funding to CRF is provided by NIDDK R01DK106188 and a NARSAD young investigator award. The author declares no conflict of interest.

\section{Carrie R Ferrario ${ }^{1}$}

${ }^{1}$ Department of Pharmacology, University of Michigan, Ann Arbor, Michigan, USA

E-mail: Ferrario@umich.edu

Brown RM, Kupchik YM, Spencer S, Garcia-Keller C Spanswick DC, Lawrence AJ et al (2015). Addiction-like synaptic impairments in dietinduced obesity. Biol Psychiatry. (e-pub ahead of print; doi:10.1016/j.biopsych.2015.11.019)

Carter A, Hendrikse J, Lee N, Yucel M, Verdejo-Garcia A, Andrews $Z$ et al (2016). The neurobiology of 'food addiction' and its implications for obesity treatment and policy. Annu Rev Nutr 36: 105-128.

Flagel SB, Akil H, Robinson TE (2009). Individual differences in the attribution of incentive salience to reward-related cues: implications for addiction. Neuropharmacology 56 Suppl 1: 139-148.

Oginsky MF, Goforth PB, Nobile CW, Lopez-Santiago LF, Ferrario CR (2016a). Eating 'junk-food' produces rapid and long-lasting increases in NAc CP-AMPA receptors; implications for enhanced cue-induced motivation and food addiction. Neuropsychopharmacology (e-pub ahead of print; doi:10.1038/ npp.2016.111).

Oginsky MF, Maust JD, Corthell JT, Ferrario CR (2016b). Enhanced cocaine-induced locomotor sensitization and intrinsic excitability of NAc 